\title{
High-frequency oscillatory ventilation: A narrative review
}

\author{
Morgan Meyers BSRC, RRT ${ }^{1}$, Nathan Rodrigues MSIS, RRT-SDS, RRT-NPS ${ }^{2}$, Arzu Ari PhD, RRT, PT, CPFT, FAARC ${ }^{2}$
}

\begin{abstract}
M Meyers, N Rodrigues, A Ari. High-frequency oscillatory ventilation: A narrative review. Can J Respir Ther 2019;55:40-46. doi: 10.29390/ cjrt-2019-004.

High-frequency oscillatory ventilation (HFOV) is a lung-protective strategy that can be utilized in the full spectrum of patient populations ranging from neonatal to adults with acute lung injury. HFOV is often utilized as a rescue strategy when conventional mechanical ventilation (CV) has failed. HFOV uses low tidal volumes and constant mean airway pressures in conjunction with high respiratory rates to provide beneficial effects on oxygenation and ventilation, while eliminating the traumatic "inflate-deflate" cycle imposed by CV. Although statistical evidence supporting HFOV is particularly low, potential benefits for its application in many clinical manifestations still remain. High-frequency oscillation is a safe and effective rescue mode of ventilation for the treatment of acute respiratory distress syndrome (ARDS). All patients who have ventilator-induced lung injury (VILI) or are at risk of developing VILI or ARDS would be suitable candidates for HFOV, especially those who have failed conventional mechanical ventilation. This narrative aims to provide a review of HFOV vis-à-vis its indications, contraindications, hazards, parameters to monitoring, patient selection, clinical goals, mechanisms of action, controls for optimizing ventilation and oxygenation, clinical application in ARDS, and a comparison with other modes of mechanical ventilation.
\end{abstract}

Key Words: high frequency oscillatory ventilation; acute respiratory distress syndrome; ventilator induced lung injury; oxygenation; clinical outcomes

\section{INTRODUCTION}

High-frequency oscillatory ventilation (HFOV) is a rescue maneuver for failed conventional mechanical ventilation. It utilizes the Taylor augmented dispersion of gases through a simple circuit in which bias flow delivers small tidal volumes for patients with acute respiratory distress syndrome (ARDS) and other medical conditions [1-4]. It prevents ventilator-induced lung injury (VILI) by reducing the risk of volutrauma, while providing adequate ventilation despite tidal volume sizes that are far below dead space [3-6]. HFOV maintains alveolar inflation at a constant, less variable airway pressure with a sinusoidal flow oscillation to prevent the lung "inflate-deflate" cycle and provides improved oxygenation [5, 7-12]. HFOV can be delivered by the Drager Babylog VN500 ventilator and Care Fusion Oscillator 3100A and 3100B ventilators [13].

Indications, contraindications, and hazards

The oscillator can be used in many clinical situations. Its benefits are valued when there is failure of conventional ventilation, when refractory hypoxemia is present and in clinical presentations comprising large air leaks or systemic circulatory problems [3, 14, 15]. However, it is essential to recognize situations in which the use of the oscillator carries the propensity to lead to deleterious consequences and patient harm. Commonly, patients receiving HFOV require a greater amount of sedation and neuromuscular blockade, which can subsequently lead to lengthier hospital stays [3, 16-19]. There are many common indications, limitations, and hazards associated with HFOV (Table 1).

Currently, HFOV is only indicated as a rescue therapy. It is beneficial for patients with severe respiratory failure who are failing conventional ventilation or when conventional ventilation settings are approaching harmful parameters, which could lead to trauma [1, 2, 9, 17, 20-22].
The mode is considered an alternative lung-protective ventilation strategy for patients with VILI and ARDS [9, 20-22]. Additionally, HFOV has been recommended as second-line therapy for the management of the ventilated patient with a bronchopleural fistula [23]. In the neonatal population, HFOV is indicated for patients with neonatal air leak syndrome, persistent pulmonary hypertension, and meconium aspiration [24-26]. In the adult population, HFOV is beneficial in air leak syndromes such as pneumothorax and pulmonary interstitial emphysema, where the lungs are difficult to keep open $[15,19,23,24,27]$.

There are no specific contraindications regarding HFOV, but there are some adverse effects. HFOV is less effective in disease processes with increased airway resistance, which can lead to air trapping and hyperinflation $[7,28]$. If this goes unmonitored, it may lead to pulmonary barotrauma $[7,9,29,30]$. This includes pneumothorax, pneumomediastinum, pneumopericardium, and pulmonary interstitial emphysema [7, 28]. The cardiovascular effects of HFOV include decreased venous return, decreased cardiac output, intraventricular hemorrhage, and increased intrathoracic pressures [11, 31]. When intubated the patient's natural defense mechanisms are removed, putting the patient at greater risk for sepsis [7].

Relative limitations associated with the use of the oscillator include the unavailability of transport ventilators and intrinsic limitations pertaining to loud or noisy machines that can inhibit the ability to perform physical examination and recognition of co-occurring hazards [1]. Although there is no oscillator manufactured specifically for transport purposes, a high-frequency percussive ventilation device, the Bronchotron, may be utilized. This pneumatic device ventilates and oxygenates as well as HFOV at equivalent ventilator settings. In some situations, it may be necessary to intermittently pause the oscillator to

\footnotetext{
${ }^{1}$ Methodist Children's Hospital, San Antonio, TX, USA

${ }^{2}$ Department of Respiratory Care, Texas State University, Round Rock, TX, USA

Correspondence: Arzu Ari, PhD, RRT, PT, CPFT, FAARC, Department of Respiratory Care, Texas State University, 200 Bobcat Way, Willow Hall \#214, Round Rock, TX 78665, USA. Tel.: +512-246-2682; E-mail: arzuari@txstate.edu orarzuari@hotmail.com
} 


\section{TABLE 1}

\section{Indications, contraindications, and hazards associated with the use of HFOV.}

Indications

- Ventilator-associated lung injury [9, 21, 22, 29]

- Alveolar hemorrhage

- Large air leak with inability to keep lungs open [15, 19, 23, 24, 27]

- Abdominal Compartment Syndrome [7]

- Failure of conventional mechanical ventilation $[5,7]$

- Refractory hypoxemia [7, 11, 19, 23, 37]

- Increased intracranial pressure [7, 38]

- Persistent pulmonary hypertension [25]

- Acute Respiratory Distress Syndrome [1, 5-7, 11, 22, 23, 25-27, 30-34]

- Pulmonary Interstitial Emphysema [26]

- Meconium aspiration [26]

- Pulmonary hypoplasia [26]

- Bronchopulmonary fistulae [23]

\section{Contraindications/hazards}

- Higher intrathoracic pressures [11]

- Right ventricular preload; require volume administration \pm inotropic support [11]

- Pneumothorax $[7,28]$

- Migration/displacement of ETT [31]

- Bronchospasm

- Airway obstruction from mucus plugging, secretions, hemorrhage, or clot $[7,28]$

- Barotrauma [4, 7, 9, 20, 21, 30, 31, 39, 40]

- Pneumomediastinum [7]

- Subcutaneous emphysema [7]

- Multiple organ failure [7]

- Sepsis [7]

- Refractory acidosis [3]

- Intraventricular hemorrhage [31]

- Cellular injury [31]

- Increased pulmonary capillary wedge pressure [31]

\section{TABLE 2}

\section{Parameters to monitor during the use of HFOV}

\begin{tabular}{ll}
\hline Oscillator & \\
\hline Mean airway pressure $[23,26,30,46]$ & $\begin{array}{l}\text { Bias flow }[4,26] \\
\text { Fraction of inspired oxygen }\left(\mathrm{F}_{\mathrm{i}} \mathrm{O}_{2}\right) \\
\text { Frequency }(\mathrm{Hz})[23,46]\end{array}$ \\
$\begin{array}{l}\text { Plarms } \\
\text { Pressure amplitude }[23,46]\end{array}$ & \\
\hline Patient & \\
\hline Oxygenation index $[23]$ & Heart rate [46] \\
$\mathrm{P}_{\mathrm{a}} \mathrm{O}_{2} / \mathrm{F}_{\mathrm{i}} \mathrm{O}_{2}[23,30]$ & Blood pressure [46] \\
Arterial pH $[23,26]$ & Alveolar-arterial oxygenation \\
& difference [46] \\
$\mathrm{P}_{\mathrm{a}} \mathrm{CO}_{2}(\mathrm{~mm} \mathrm{Hg})[13,23,46]$ & Chest x-Ray [46, 47] \\
$\mathrm{P}_{\mathrm{a}} \mathrm{O}_{2}(\mathrm{~mm} \mathrm{Hg})[23,26,46,47]$ & Right ventricular load [30] \\
$\mathrm{SpO}_{2}[23,26]$ & Transpulmonary pressure [30] \\
\hline
\end{tabular}

perform a patient assessment, especially cardiac status. This should be performed minimally and with physician approval. Clinicians should closely and continually monitor the patient's clinical presentation and be able to recognize the potentially deleterious effects associated with utilizing this unique strategy of positive pressure ventilation.

An additional limitation associated with HFOV is alveolar derecruitment precipitated by suctioning. Suctioning the patient receiving HFOV may be beneficial to ensure patency of the endotracheal tube (ETT); however, it should be used in moderation, as disconnecting the patient from the oscillator leads to alveolar derecruitment [27]. Suctioning should only be preformed per organizational policy or when clinically deemed necessary. Suctioning may be indicated when chest wiggle is decreased or absent, when a mucus plug or secretions are suspected, if there is a decrease in $\mathrm{SpO}_{2}$ or transcutaneous $\mathrm{O}_{2}$ level, or when there is an increase in the transcutaneous $\mathrm{CO}_{2}$ levels $[3,32]$. The deleterious effects caused by suctioning may be minimized, but not fully eliminated, by utilizing a closed suction system [32]. A sustained inflation recruitment maneuver may be necessary following suctioning to compensate for the prior period of derecruitment [33-35]. To prevent derecruitment, once the patient is on the oscillator the patient should not be disconnected from the oscillator unless there is no other option [36].

\section{Parameters to monitor}

Many variables must be closely monitored during the use of HFOV (Table 2). Not only is it important to monitor the oscillator, but also the patient's clinical appearance [41]. An essential tool available to clinicians for the assessment of the patient's response to HFOV is the arterial blood gas (ABG) [42]. An ABG should be obtained 15-30 minutes following the initiation of $\mathrm{HFOV}$ to determine $\mathrm{PaCO}_{2}$ trending $[43,44]$.
Subsequent ABGs should generally be obtained at 30- to 60-minute intervals, until stabilization occurs $[44,45]$. Once the patient is stabilized, and there are minimal changes occurring with the oscillator settings, ABGs can then subsequently be obtained every 6 hours $[44,45]$.

During oscillator use, minute ventilation and tidal volume are affected by several factors including pressure amplitude, frequency, ETT size, amount of cuff leak, and patient lung characteristics [4, 33, 41]. To properly set a $5 \mathrm{cmH}_{2} \mathrm{O}$ cuff leak, air should carefully be withdrawn from the ETT pilot balloon using a syringe, until mean airway pressure (mPaw) drops $5 \mathrm{cmH}_{2} \mathrm{O}$ below the set mPaw. Afterwards, mPaw should be increased back to the previous setting [1]. On the oscillator machine, monitored parameters include the settings and alarms. While the patient is receiving HFOV via oscillator, clinicians must pay close attention to more than just their vital signs such as heart rate blood pressure, and $\mathrm{S}_{\mathrm{p}} \mathrm{O}_{2}$. Lab values are equivalently significant, as they provide valuable information to assist the clinician in making precise changes to either escalate or wean oscillator settings accordingly.

\section{Patient selection}

Use of HFOV via oscillator is typically limited to clinical situations in which the patient is in an acute, critical, poor state of health. In the adult and pediatric population, this can mean that they have suffered an unfortunate trauma or are undergoing a major health crisis $[3,7,8$, 24,31 ]. In the infant population, this is also due to unfortunate conditions in which there is meconium aspiration or pulmonary interstitial emphysema [7, 8, 24, 31]. All patients who have VILI or are at risk of developing VILI or ARDS, would be suitable candidates for HFOV $[1,3-5,7,8,13,14,18,22,26,27,37,38]$. Variations may be necessary based on clinical disease state and patient population. Examples of this include air leaks, fluid resuscitation to keep central venous pressure between 8 and $12 \mathrm{~mm} \mathrm{Hg}$, and the presence of radiologically apparent large and multiple cysts in the lungs $[15,26,30]$.

\section{Clinical goals}

The primary goal for use of HFOV via oscillator is to limit lung injury and improve clinical outcome. HFOV utilizes active inspiratory and expiratory phases to produce small tidal volumes, usually equal to or less than dead space $[4,5,48]$. The rapid respiratory rate helps maintain alveolar ventilation, while the lungs maintain inflation through a constant mPaw $[5,7$, $11,13,23,26,39]$. HFOV delivers reasonable oxygenation to limit oxygen toxicity $[7,11,19,46]$. This method uses permissive hypercapnia to provide ventilatory support and maintain normal cellular function, allowing $\mathrm{PaCO}_{2}$ to rise while maintaining an arterial $\mathrm{pH}$ between 7.25 and 7.30 . This strategy minimizes VILI, reduces the incidence of secondary chronic lung disease, improves ventilation/perfusion $(\mathrm{V} / \mathrm{Q})$ mismatch, does not impair cardiac output, and improves lung recruitment without overdistension $[1,4,5,9,11,13,17,18,25,26,30,31,34,40,42]$. 


\section{Mechanisms of action}

Use of positive pressure ventilation carries the propensity to damage the lungs. During CV there are large fluctuations in the zones of injury resultant from the cycle changes between the inspiration and expiration phases, leading to significant damage. During HFOV, the entire cycle operates in the "safe window" to achieve homogeneous aeration of the lung and avoid the injury zones [10, 11, 24, 47]. Injury can occur at both ends of the safe window [49]. On the upper end, in the zone of overdistension, injury occurs from mechanical disruption, surfactant dehydration, and fluid accumulation [49]. On the lower end, in the zone of derecruitment, injury occurs due to atelectrauma and biotrauma [49]. HFOV works within the safe window allowing the recruitment of collapsed alveoli and preventing atelectasis [50]. This is achieved through the use of rapid, tiny oscillations produced by a reciprocating piston $[1,5,9,10]$. Both inspiration and expiration are active processes secondary to the piston striking forward creating positive pressure in the airway and then recoiling back, generating negative pressure [5]. The amplitude of the wave, which is set by the power control, determines the forward and backward excursion of the piston and helps determine the tidal volume [9]. Tiny tidal volumes are pushed in and out of the lungs, oscillating around a set pressure, hence the name of the unit-the oscillator [51]. A rigid ventilator circuit with low compliance is used in HFOV.

\section{Optimizing oxygenation}

The primary variables in achieving optimal oxygenation are mPaw and $\mathrm{F}_{\mathrm{i}} \mathrm{O}_{2}$ [3]. Mean airway pressure is a pressure used to optimize lung volume and, thus, to increase the alveolar surface area for gas exchange [5, 8, 51]. In turn, this directly affects the $\mathrm{PaO}_{2}[1,8,31]$. Mean airway pressure should be utilized to recruit atelectatic alveoli while preventing derecruitment. Although the lung must be recruited, this must be done conservatively to avoid overdistension. Alveolar atelectasis or overdistension can result in an increase in pulmonary vascular resistance. To initially introduce mPaw, it should be set $3-5 \mathrm{~cm} \mathrm{H}_{2} \mathrm{O}$ above the corresponding mPaw used prior during conventional ventilation [5]. mPaw normal ranges are $25-30 \mathrm{~cm} \mathrm{H}_{2} \mathrm{O}$, with a maximum of $45-60 \mathrm{~cm}$ $\mathrm{H}_{2} \mathrm{O}$ [1]. Adequate lung expansion in adults is accomplished by the visualization of the ninth posterior rib above the level of the diaphragm. As mPaw increases, the volume in the lung increases and the patient's diaphragm is displaced downward; placement of the diaphragm via chest x-ray usually correlates to an acceptable $\mathrm{P}_{2} \mathrm{O}_{2}$. The mPaw should not be reduced during the first 24 hours to permit an adequate amount of time for alveolar recruitment. The $\mathrm{FiO}_{2}$ is titrated by a blender attached to the oscillator $[1,5]$. If the patient is overoxygenated, the $\mathrm{FiO}_{2}$ and or mPaw should be decreased. If the patient's $\mathrm{P}_{\mathrm{a}} \mathrm{O}_{2}$ is low, the $\mathrm{FiO}_{2}$ and mPaw should be increased to increase oxygenation. Figure 1 depicts oxygenation controls on the HFOV.

\section{Optimizing ventilation}

The primary variables in ventilation are tidal volume, chest wiggle, and frequency [26]. Amplitude can be adjusted by the power control [1]. Adjusting the power control regulates the amount of piston displacement by changing the amount of power going to the piston and the backward-forward movement of the piston. The degree of deflection of the piston (amplitude) determines the tidal volume. As the amplitude increases, the pressure gradient increases and, therefore, the tidal volume delivered to the patient increases [5]. The increase in amplitude depends on the resistance the piston must work with to move forward [36]. If the patient has low lung compliance the piston must work against a greater pressure resulting in less change in tidal volume. The same can be said for a patient with high airway resistance [36]. If the patient is underventilated and the $\mathrm{PaCO}_{2}$ level is high, the amplitude should be increased to blow off more $\mathrm{PaCO}_{2}$. If the patient is overventilated and the $\mathrm{PaCO}_{2}$ level is low, the amplitude should be decreased to allow less $\mathrm{PaCO}_{2}$ to be blown off. To set the amplitude, an ABG should be obtained on conventional ventilation, and 20 should be added to the $\mathrm{PaCO}_{2}$ level. The normal amplitude is set between 20 and 30 in neonates, with settings up to $90 \mathrm{~cm} \mathrm{H}_{2} \mathrm{O}$ possible. Appropriateness of the power setting is determined by observing the chest wiggle factor (CWF) $[1,5,8]$. Chest wiggle should be visible from the level from the clavicles to the mid-thigh or the eigth or ninth rib [4]. Increased amplitude will generate an increased chest wiggle. Chest wiggle should always be reassessed after positional changes. If CWF is diminished or absent, consider decreased pulmonary compliance, ETT disconnect, ETT obstruction, or severe bronchospasm [36]. If the CWF is unilateral, consider ETT displacement (right mainstem), or pneumothorax [36]. Clinicians must be proactive and monitor patients closely during the use of HFOV via oscillator.

In HFOV frequency is derived from hertz that controls the time allowed for the piston to move forward and backward. There is a resonance frequency of the lungs in which optimal ventilation, through $\mathrm{CO}_{2}$ removal, occurs. Resonance frequency varies based on lung size, the degree of lung injury, amount of lung function, type and extent of the disease state, and the size of the patient. Gas transport becomes dependent on tissue inertia, rather than elastance. If there is a disturbance of normal gas flow, V/Q mismatch exacerbated by hypoxemia and worsening respiratory acidosis will occur. To overcome the effects of the resonance frequency, clinicians can optimize ventilation by providing the

\section{FIGURE 1}

Care Fusion Oscillator 3100A: depicting oxygenation controls. (A) Mean airway pressure limit knob and (B) mean airway pressure adjustment knob. Note: the $3100 \mathrm{~B}$ does not have a mean airway pressure limit knob; the monitor is located in the top left corner.

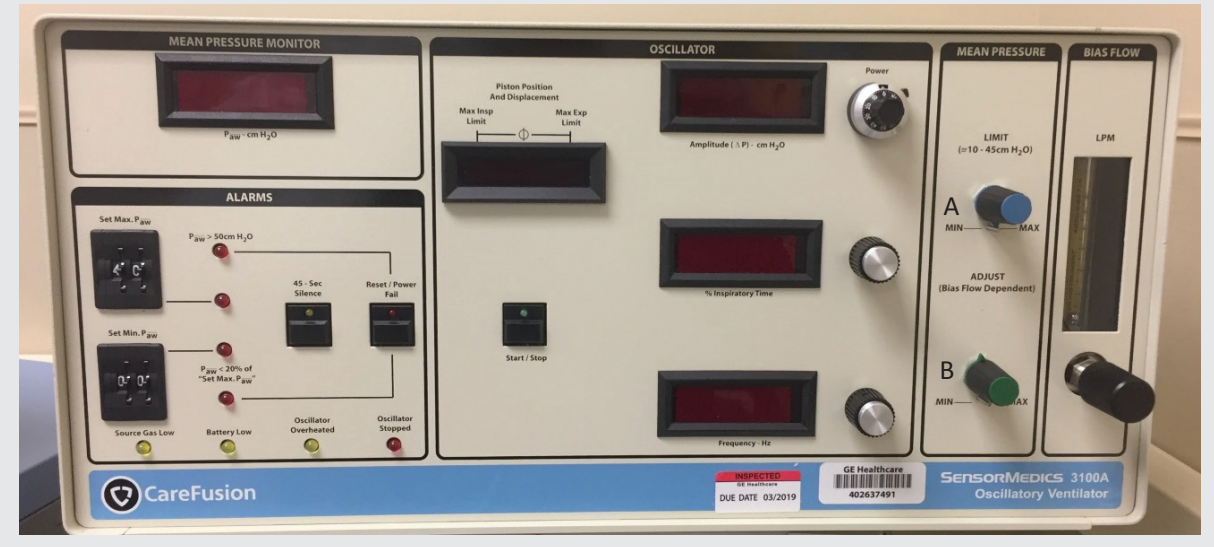


correct frequency. One hertz is 60 breaths per minute [4]. The range of hertz is 3-15 Hz, with typical initial settings of $5-6 \mathrm{~Hz}[4,5,8,33]$. Reducing the frequency causes greater volume displacement, resulting in a greater tidal volume and subsequent minute ventilation. In conventional mechanical ventilation, the respiratory rate is increased to blow off $\mathrm{CO}_{2}$, but HFOV operates in opposition to this. To decrease the $\mathrm{P}_{\mathrm{a}} \mathrm{CO}_{2}$, the frequency should be decreased. If the patient is overventilated, the frequency should be increased. Figure 2 shows ventilation controls on the HFOV.

\section{Other controls}

Although controls relating to oxygenation and ventilation are of vital importance to managing successful outcomes in HFOV, there are other controls of similar importance. Bias flow (BF) controls and indicates the rate of continuous flow of humidified blended gas through the circuit [4, 52]. The control knob is a 15 -turn pneumatic valve, which increases flow when turned clockwise. The rate of flow is indicated by a ball float and ranges from $0-60 \mathrm{~L}$ per minute in $5 \mathrm{~L}$ per minute increments $[1,4,5]$. BF is typically the first parameter set by the clinician, because it is part of the oscillator peruse calibration. If the BF is set too low, it may result in increased $\mathrm{P}_{\mathrm{a}} \mathrm{CO}_{2}$ secondary to inadequate circuit washout [52]. If the $\mathrm{BF}$ is set too high, it may inhibit $\mathrm{CO}_{2}$ elimination. On the contrary, an increase bias gas flow will increase airway pressure, thus improving oxygenation [36]. Inspiratory time \% (IT\%) represents the portion of the respiratory cycle that the piston spends in forward motion $[1,5,8,9]$. It may assist in $\mathrm{CO}_{2}$ elimination, although to a lesser extent than amplitude and frequency. The initial setting is 33\% (inspiratory:expiratory ratio $1: 2)[1,4,9]$. For some patients, an IT\% of $50 \%$ may improve ventilation and lung recruitment, but it is generally maintained at 33\% [1].

Amplitude, frequency, and inspiratory time are commonly adjusted during HFOV to increase gas displacement and improve $\mathrm{CO}_{2}$ elimination, but piston-position adjustments can be viewed as an additional tool in the management of patients requiring HFOV via oscillator [53]. More optimal placement of the piston may allow for increased $\mathrm{CO}_{2}$ elimination during HFOV [53]. This is especially important in the premature population, as a slight deviation of the piston to either side can cause $\mathrm{CO}_{2}$ retention, which could be detrimental.

It is essential for the alarms to be set properly during the use of HFOV via oscillator to monitor the patient's clinical status. There are many factors that can affect the mPaw. In spontaneously breathing patients the clinical status of the patient, sedation level, or insufficient bias flow rate can all affect the mPaw [54]. In this case, it may be necessary to readjust the mPaw. The high-pressure alarm should be set $3 \mathrm{~cm} \mathrm{H}_{2} \mathrm{O}$ to $5 \mathrm{~cm} \mathrm{H}_{2} \mathrm{O}$ above the set mPaw. It is best practice to consult the institutional protocol for HFOV alarms, as this alarm limit value will vary accordingly. If there is an obstruction on the expiratory limb or in the pressure sensing line, the high-pressure alarm will sound, and the patient circuit must be replaced [54]. Lastly, if the patient circuit temperature rises, the mPaw may be affected, so the circuit temperature must be maintained accordingly [54]. The low-pressure alarm should be set $5 \mathrm{~cm} \mathrm{H} \mathrm{H}_{2} \mathrm{O}$ below the set mPaw. The mPaw will be low if there is a leak in the humidifier or patient circuit, which will require the leak to be fixed [54]. The patient circuit leak is most commonly noted from the cap diaphragm [54]. If the water trap stopcock is open, the mPaw will trigger alarm as low, and the stopcock must be closed to resolve the issue [54]. Figure 3 shows other important controls such as bias flow, inspiratory time percent, alarms, and piston position and displacement.

\section{HFOV and ARDS}

Although medically necessary, mechanical ventilation can potentiate lung damage through a variety of mechanisms collectively referred to as VILI [55]. Traditionally, VILI contributed to mechanical stress and strain within the lungs, as the mechanical force applied to the pulmonary epithelium lining the airway and the alveoli initiates a resultant inflammatory response within the lungs $[22,47]$. This can spread to other organs causing biotrauma [47]. To combat this, the Acute Respiratory Distress Syndrome Network (ARDSnet) found that mortality can be significantly reduced through the use of lower tidal volumes for patients with ARDS [23,56]. HFOV is the ideal technique to achieve this. HFOV generates a tidal volume that is less than dead space $(1-3 \mathrm{~mL} / \mathrm{kg})$ in conjunction with high positive end expiratory pressure (PEEP) $[4,22,30]$. Low tidal volumes have been associated with improved outcome because it avoids the cyclic opening and closing of collapsed alveoli applied during each tidal breath $[1,4,11,57]$. HFOV works to recruit noncompliant, atelectatic regions of the lungs, thus providing a subsequent increase in overall lung volume [47]. With the use of the oscillator, there is less pulmonary and systemic inflammation, less histologic evidence of lung injury, and lower mortality [7].

\section{HFOV vs. APRV}

Modern day technology has advanced ventilator management, yet $31 \%-38 \%$ of ARDS patients die from respiratory failure [36]. Secondary to this, alternative rescue modes of mechanical ventilation have been developed, including airway pressure release ventilation (APRV) and HFOV [36]. Both of these methods work to improve oxygenation of the lungs by keeping them inflated for an extended period of time, also known as the open-lung concept [36]. These modes should be considered when a patient's $\mathrm{F}_{\mathrm{i}} \mathrm{O}_{2}$ is greater than $60 \%$, PEEP is greater than $15 \mathrm{~cm} \mathrm{H}_{2} \mathrm{O}$, and plateau pressures are greater than $30 \mathrm{~cm} \mathrm{H}_{2} \mathrm{O}$ [36].

\section{FIGURE 2}

Care Fusion Oscillator 3100A: depicting ventilation controls. (A) Power control (amplitude) and (B) frequency (hertz).

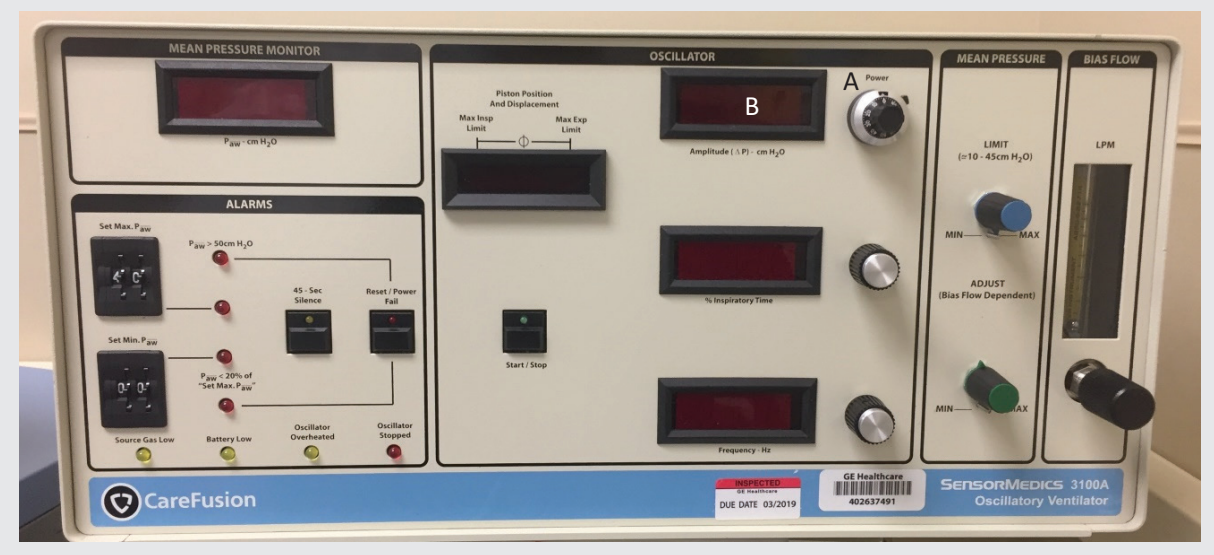




\section{FIGURE 3}

Care Fusion Oscillator 3100A: depicting other important controls. (A) bias flow, (B) inspiratory time percent, (C) piston position and displacement, and (D) alarms.

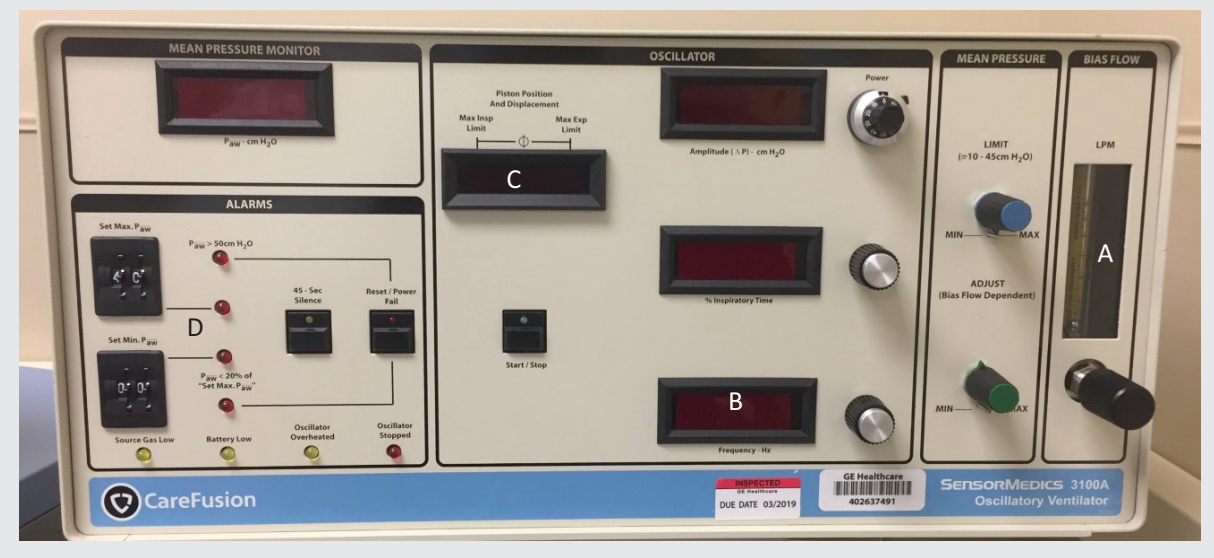

Although the mortality benefit of these modes has not been consistent, these modes may be beneficial for certain patient populations [36].

$\mathrm{APRV}$ is a rescue strategy for failure of $\mathrm{CV}$, which provides increased airway pressures to facilitate alveolar recruitment [23, 24, 36, 58]. APRV is an alternative method to the ARDSnet approach. This mode is designed to promote oxygenation by providing continuous positive airway pressure (CPAP) with intermittent releases of airway pressure [36]. Ventilatory support is determined by the two CPAP levels, pressure high $\left(\mathrm{P}_{\text {high }}\right)$ and pressure low $\left(\mathrm{P}_{\text {low }}\right)$ [36]. $\mathrm{P}_{\text {low }}$ is generally set at zero, otherwise known as zeep, so the tidal volume is dependent on the $\mathrm{P}_{\text {high }}$ [36]. When compared with conventional ventilation, APRV is associated with significantly lower peak and plateau airway pressures for a given tidal volume [36]. In ARDS patients, more compliant regions of the lungs receive more flow and fibrotic alveoli collapses earlier during exhalation [36]. Based off of this, APRV has the potential to cause volutrauma on inhalation and atelectrauma on exhalation [36]. One of the largest benefits of APRV is that it allows spontaneous breathing, facilitating a lower level of required sedation [1]. Patient interaction is essential in APRV, so the use of paralysis is not necessary [36]. APRV has been associated with a reduction in ICU length of stay and duration of mechanical ventilation, but studies have failed to show consistent reduction in ARDS-related mortality $[1,36]$.

HFOV delivers small tidal volumes at a constant mean airway pressure $[1,12,23]$. This strategy minimizes barotrauma, volutrauma, and atelectrauma [1, 4, 12, 29, 31, 39, 47]. HFOV maximizes alveolar recruitment thus improving oxygenation. Both HFOV and APRV improve oxygenation in patients with ARDS but there are contradicting opinions regarding the use of HFOV in comparison with APRV [36]. Some believe that HFOV reduces the risk of further lung injury, leading to maximal lung recruitment and minimal overdistension [47, 48, 57]. Others believe that like APRV, HFOV has failed to show consistent reductions in ARDS-related mortality [14, 40, 47, 58-61]. A major difference in the two modes is that APRV allows spontaneous ventilation, whereas HFOV does not [36]. Thus APRV is associated with decreased amounts of sedation and decreased number of ventilator days [36]. On the other hand, HFOV keeps the lungs open at all times, running less risk of VILI [36]. An additional advantage of APRV is that its ventilators are battery powered and can be used for patients who require transportation to imaging, operations, etc. Further comparison studies between the two modes would be beneficial [36].

\section{HFOV vs. CV}

Charlie Bryan, a physiologist and pediatric intensivist, stumbled upon what later became known as HFOV while performing a study using forced oscillations to examine the effects of neuromuscular blockers on the lungs [47]. Since then, there have been several large multicenter randomized trials to compare HFOV and CV regarding their impact in ARDS [29-31, 47, 62-64]. In the area of HFOV, these studies generally did not result in positive patient outcomes. In fact, the first multicenter randomized trial of HFOV in 1989 was stopped early because of high rates of intraventricular hemorrhage [31].

The OSCILLATE trial enrolled adults with moderate to severe ARDS in ICUs in several different countries [47]. According to the patient's severity of hypoxemia, the HFOV protocol was applied using recruitment maneuvers and high mPaw [33, 41, 47]. The CV protocol used low tidal volumes [47]. The OSCILLATE trial found that high mPaw applied by HFOV significantly impaired hemodynamics, including cardiac output, and may have contributed to worsening conditions $[22,47]$. The study was stopped early secondary to mortality in the HFOV group being significantly higher than in the CV group [47, 59, 65].

The OSCAR trial enrolled patients with severe ARDS in the United Kingdom [47]. A complex algorithm was used to titrate HFOV, with goals to improve lung recruitment for oxygenation while reducing frequency as needed to improve $\mathrm{CO}_{2}$ clearance [47]. Unlike OSCILLATE, OSCAR used lower PEEP in the CV protocol. This study found no difference in mortality between HFOV and CMV [22, 47, 65].

The RESTORE study found the use of HFOV in ARDS patients required an increased amount sedation and length of hospital stay, yielding an overall negative impact on ARDS [47]. Contrary to these studies, HFOV is a great strategy for improving oxygenation. A meta-analysis found an interaction between baseline $\mathrm{P}_{\mathrm{a}} \mathrm{O}_{2} / \mathrm{F}_{\mathrm{i}} \mathrm{O}_{2}$ and the effect of HFOV, with increasing harm when HFOV is used with higher $\mathrm{P}_{\mathrm{a}} \mathrm{O}_{2} / \mathrm{F}_{\mathrm{i}} \mathrm{O}_{2}$ values [60]. The exact value at which $\mathrm{HFOV}$ changes from harm to benefit is uncertain [60]. This suggests that HFOV should not be used for patients with mild or moderate ARDS, but still remains a potential modality for patients with severe hypoxia with ARDS [60]. Overall, this study concluded that HFOV might improve survival of patients with severe hypoxemia while on conventional mechanical ventilation, especially when alternative approaches such as proning or extracorporeal membrane oxygenation are unavailable [60]. An ongoing study, $\mathrm{EPOCH}$, is comparing a strategy of preventing atelectrauma with a transpulmonary pressure of $0 \mathrm{cmH}_{2} \mathrm{O}$ at end expiration with transpulmonary pressure of 15 using CV and HFOV [47].

Despite the less than impressive numbers in reference to ARDS there still remain clinical situations where HFOV could prove beneficial [14, $47,61,65]$. 


\section{SUMMARY}

HFOV maximizes alveolar recruitment and is a great tool in alveolar recruitment that can be beneficial in preventing lung injury. The strategy utilizes volumes that are less than dead space, while providing a constant mPaw to maintain alveolar recruitment thereby preventing VILI. HFOV maintains lung inflation at a constant; less variable airway pressure with a sinusoidal flow oscillation to prevent the lung inflate-deflate cycle and provides improved oxygenation. To optimize oxygenation, manipulate the mean airway pressure and $\mathrm{FiO}_{2}$. To optimize ventilation, manipulate the amplitude, chest wiggle, and $\mathrm{Hz}$. It is necessary for clinicians to assess patients noting chest wiggle, endotracheal tube placement, and provide therapy or adjustment of settings when necessary based on the findings. ARDS results in severe morbidity and mortality. HFOV is an alternative solution to combat ARDS through the use of active inspiratory and expiratory phases and small tidal volumes at a constant mPaw to limit additional injury to the lung while working in the safe window. This maximizes alveolar recruitment and improves oxygenation. Reducing the cyclic opening and closing of alveoli leads to a reduction in inflammatory markers. APRV also utilizes the safe window approach by limiting overdistention and using high constant positive pressure to recruit alveoli. In this mode, patients can breathe spontaneously, and require less sedation. There is no evidence supporting APRV or HFOV in relation to mortality and morbidity.

\section{Conflict of interest}

AA discloses relationship with the CHEST Foundation, Bayer Pharmaceuticals, Aerogen Ltd, ARC Medical and Sunovion Pharmaceuticals. NR and MM have no conflict of interest with regards to this article.

\section{REFERENCES}

1. Facchin F, Fan E. Airway pressure release ventilation and high-frequency oscillatory ventilation: Potential strategies to treat severe hypoxemia and prevent ventilator-induced lung injury. Respir Care 2015;60(10): 1509-21. doi: 10.4187/respcare.04255.

2. Pillow JJ. High-frequency oscillatory ventilation: Mechanisms of gas exchange and lung mechanics. Crit Care Med 2005;33(3): S135-41. doi: 10.1097/01.CCM.0000155789.52984.B7.

3. Jarvis S, Burt MK, English W. High frequency oscillatory ventilation. Anaesthesia Tutorial of the Week. 2012;261: 1-11.

4. Hupp SR, Turner DA, Rehder KJ. Is there still a role for high-frequency oscillatory ventilation in neonates, children and adults? Expert Rev Respir Med 2015;9(5): 603-18. doi: 10.1586/17476348.2015.1077119.

5. Bouchut J-C, Godard J, Claris O. High-frequency oscillatory ventilation. Anesthesiol J Am Soc Anesthesiol 2004;100(4): 1007-12. doi: 10.1097/ 00000542-200404000-00035.

6. Vento G, Matassa PG, Ameglio F, et al. HFOV in premature neonates: Effects on pulmonary mechanics and epithelial lining fluid cytokines. A randomized controlled trial. Intensive Care Med 2005;31(3): 463-70. doi: 10.1007/s00134-005-2556-x.

7. Briggs S, Goettler CE, Schenarts PJ, et al. High-frequency oscillatory ventilation as a rescue therapy for adult trauma patients. Am J Crit Care 2009;18(2): 144-8. doi: 10.4037/ajcc2009303.

8. Bryan A, Bohn D. High frequency oscillatory ventilation. Can Respir J 1996;3(6): 368-9. doi:10.1007/s001340051099

9. Clark RH, Null DM, Gerstmann DR. Prospective randomized comparison of high-frequency oscillatory and conventional ventilation in respiratory distress syndrome. Pediatrics 1992;89(1): 5-12.

10. Courtney SE, Durand DJ, Asselin JM, et al. High-frequency oscillatory ventilation as a rescue therapy for adult trauma patients. J Perinatol 1992;100(4): 643-52. doi:10.1007/s001340051099

11. Squires KAG, De Paoli AG, Williams C, Dargaville PA. High-frequency oscillatory ventilation with low oscillatory frequency in pulmonary interstitial emphysema. Neonatology 2013;104(4): 243-9. doi: 10.1159/ 000353376.

12. Singh JM, Stewart TE. High-frequency mechanical ventilation principles and practices in the era of lung-protective ventilation strategies. Respir Care Clin N Am 2002;8(2): 247-60. doi: 10.1016/S1078-5337(02) 00007-2.

13. Fan E, Brodie D, Slutsky AS. Acute respiratory distress syndrome: Advances in diagnosis and treatment. Jama 2018;319(7): 698-710. doi: 10.1001/jama.2017.21907.
14. Ng J, Ferguson ND. High-frequency oscillatory ventilation: Still a role? Curr Opin Crit Care 2017;23(2): 175-9. doi: 10.1097/MCC.000000000 0000387.

15. Aurilia C, Ricci C, Tana M, et al. Management of pneumothorax in hemodynamically stable preterm infants using high frequency oscillatory ventilation: Report of five cases. Ital J Pediatr 2017;43(1): 114. doi: $10.1186 / \mathrm{s} 13052-017-0436-\mathrm{y}$.

16. Sessler CN. Sedation, analgesia, and neuromuscular blockade for high-frequency oscillatory ventilation. Crit Care Med 2005;33(3): S209-16. doi: 10.1097/01.CCM.0000156794.96880.DF.

17. Fessler HE, Hess DR. Respiratory controversies in the critical care setting. Does high-frequency ventilation offer benefits over conventional ventilation in adult patients with acute respiratory distress syndrome? Respir Care 2007;52(5): 595-605

18. Burry LD, Seto K, Rose L, Lapinsky SC, Mehta S. Use of sedation and neuromuscular blockers in critically ill adults receiving highfrequency oscillatory ventilation. Ann Pharmacother 2013;47(9): 1122-9. doi: $10.1177 / 1060028013503121$.

19. Gupta P, Green JW, Tang X, et al. Comparison of high-frequency oscillatory ventilation and conventional mechanical ventilation in pediatric respiratory failure. JAMA Pediatr 2014;168(3): 243-9. doi: 10.1001/ jamapediatrics.2013.4463.

20. Derdak S. High-frequency oscillatory ventilation for acute respiratory distress syndrome in adult patients. Crit Care Med 2003;31(4): S317-23. doi: 10.1097/01.CCM.0000057910.50618.EB.

21. Dreyfuss D, Gaudry S. Might high-frequency oscillatory ventilation improve the prognosis of more severe acute respiratory distress syndrome? Not so sure. Am J Respir Crit Care Med 2018;197(6): 838-9. doi: 10.1164/rccm.201709-1927LE.

22. Vincent J-L. High-frequency oscillation in acute respiratory distress syndrome. The end of the story? Am J Respir Crit Care Med 2017;196(6): 670-1. doi: 10.1164/rccm.201703-0475ED.

23. Vitale D, Patrizio Petrone MD, Marini CP. High-Frequency Oscillatory Ventilation (HFOV) as primary ventilator strategy in the management of severe acute respiratory distress syndrome (ARDS) with Pneumothorax in the Setting of Trauma. Am Surg 2017;83(3): E99.

24. Rettig JS, Smallwood CD, Walsh BK, et al. High-frequency oscillatory ventilation in pediatric acute lung injury: A multicenter international experience. Crit Care Med 2015;43(12): 2660-7. doi: 10.1097/ CCM.00000000000001278.

25. Coates EW, Klinepeter ME, O'shea TM. Neonatal pulmonary hypertension treated with inhaled nitric oxide and high-frequency ventilation. J Perinatol 2008;28(10): 675. doi: 10.1038/jp.2008.76.

26. Imai Y, Nakagawa S, Ito Y, Kawano T, Slutsky AS, Miyasaka K. Comparison of lung protection strategies using conventional and high-frequency oscillatory ventilation. J Appl Physiol 2001;91(4): 1836-44. doi: 10.1152/jappl.2001.91.4.1836.

27. Johnson AH, Peacock JL, Greenough A, et al. High-frequency oscillatory ventilation for the prevention of chronic lung disease of prematurity. N Engl J Med 2002;347(9): 633-42. doi: 10.1056/NEJMoa020432.

28. Mehta S, Lapinsky SE, Hallett DC, et al. Prospective trial of high-frequency oscillation in adults with acute respiratory distress syndrome. Crit Care Med 2001;29(7): 1360-9. doi: 10.1097/00003246-200107000-00011.

29. Derdak S, Mehta S, Stewart TE, et al. High-frequency oscillatory ventilation for acute respiratory distress syndrome in adults: A randomized, controlled trial. Am J Respir Crit Care Med 2002;166(6): 801-8. doi: $10.1164 / \mathrm{rccm} .2108052$.

30. Samransamruajkit R, Rassameehirun C, Pongsanon K, et al. A comparison of clinical efficacy between high frequency oscillatory ventilation and conventional ventilation with lung volume recruitment in pediatric acute respiratory distress syndrome: A randomized controlled trial. Indian J Crit care Med 2016;20(2): 72. doi: 10.4103/0972-5229.175940.

31. Bollen CW, van Well GTJ, Sherry T, et al. High frequency oscillatory ventilation compared with conventional mechanical ventilation in adult respiratory distress syndrome: A randomized controlled trial [ISRCTN24242669]. Crit Care 2005;9(4): R430. doi: 10.1186/cc3737.

32. Care AA for R. Endotracheal suctioning of mechanically ventilated patients with artificial airways 2010. Respir Care 2010;55(6): 758-64.

33. Fessler HE, Derdak S, Ferguson ND, et al. A protocol for high-frequency oscillatory ventilation in adults: Results from a roundtable discussion. Crit Care Med 2007;35(7): 1649-54. doi: 10.1097/01.CCM.0000269026.40739.2E.

34. Cartotto R, Walia G, Ellis S, Fowler R. Oscillation after inhalation: High frequency oscillatory ventilation in burn patients with the acute respiratory distress syndrome and co-existing smoke inhalation injury. J Burn Care Res 2009;30(1): 119-27. doi: 10.1097/BCR.0b013e3181920fe6. 
35. Ferguson ND, Chiche J-D, Kacmarek RM, et al. Combining highfrequency oscillatory ventilation and recruitment maneuvers in adults with early acute respiratory distress syndrome: The Treatment with Oscillation and an Open Lung Strategy (TOOLS) Trial pilot study. Crit Care Med 2005;33(3): 479-86. doi: 10.1097/01.CCM.0000155785. 23200.9E.

36. Stawicki SP, Goyal M, Sarani B. Analytic reviews: High-frequency oscillatory ventilation (HFOV) and airway pressure release ventilation (APRV): A practical guide. J Intensive Care Med 2009;24(4): 215-29. doi: $10.1177 / 0885066609335728$.

37. Miyaji M, Baines T, Samraj RS. 1163: High-frequency oscillatory ventilation as a rescue mode for refractory hypercarbia in children. Crit Care Med 2018;46(1): 565. doi: 10.1097/01.ccm.0000529168.98474.60.

38. Bennett SS, Graffagnino C, Borel CO, James ML. Use of high frequency oscillatory ventilation (HFOV) in neurocritical care patients. Neurocrit Care 2007;7(3): 221-6. doi: 10.1007/s12028-007-0084-y.

39. Zannin E, Dellaca RL, Dognini G, et al. Effect of frequency on pressure cost of ventilation and gas exchange in newborns receiving highfrequency oscillatory ventilation. Pediatr Res 2017;82(6): 994. doi: 10.1038/pr.2017.151.

40. Gu X, Wu G, Yao Y, Shi D, Song Y. Is high-frequency oscillatory ventilation more effective and safer than conventional protective ventilation in adult acute respiratory distress syndrome patients? A meta-analysis of randomized controlled trials. Crit Care 2014;18(3): R111. doi: 10.1186/ cc13900.

41. Higgins J, Estetter B, Holland D, Smith B, Derdak S. High-frequency oscillatory ventilation in adults: Respiratory therapy issues. Crit Care Med 2005;33(3): S196-203. doi: 10.1097/01.CCM.0000155922. 78943.2D

42. Lall R, Hamilton P, Young D, Hulme C, Hall P, Shah S, et al. A randomised controlled trial and cost-effectiveness analysis of high-frequency oscillatory ventilation against conventional artificial ventilation for adults with acute respiratory distress syndrome. The OSCAR (OSCillation in ARDS) study. Health Technol Assess 2015;19(23).

43. Poddutoor PK, Chirla DK, Sachane K, Shaik FAR, Venkatlakshmi A. Rescue high frequency oscillation in neonates with acute respiratory failure. Indian Pediatr 2011;48(6): 467-70. doi: 10.1007/s13312-0110073-2.

44. Hamel DS, Cheifetz IM. High-frequency oscillatory ventilation - A clinical approach. S Afr J Crit Care 2005;21(1): 15-24.

45. APPENDIX VI HFOV Quick Guide. Augusta University Portal and Web Services, Agusta, GA. Available at https://paws.augusta.edu/pub/respiratory/Documents/adult_protocol/MechanicalVentappendixVI.pdf Accessed on November 7, 2018.

46. Bateman ST, Borasino S, Asaro LA, et al. Early high-frequency oscillatory ventilation in pediatric acute respiratory failure. a propensity score analysis. Am J Respir Crit Care Med 2016;193(5): 495-503. doi: 10.1164/ rccm.201507-1381OC.

47. Sklar MC, Fan E, Goligher EC. High-Frequency oscillatory ventilation in adults with ARDS: Past, present, and future. Chest 2017;152(6): 1306-17. doi: 10.1016/j.chest.2017.06.025.

48. Ethawi YH, Abou Mehrem A, Minski J, Ruth CA, Davis PG. High frequency jet ventilation versus high frequency oscillatory ventilation for pulmonary dysfunction in preterm infants. Cochrane Database Syst Rev 2016;5: CD010548. doi:10.1002/14651858.CD010548.pub2.

49. Tobin MJ. Principles and practice of mechanical ventilation. Shock 2006;26(4): 426. doi: 10.1097/01.shk.0000245023.16612.dd.
50. Ali S, Ferguson ND. High-Frequency Oscillatory Ventilation in ALI/ARDS. Critical Care Clinics. 2011;27: 487-99. doi: 10.1016/j.ccc. 2011.04.006.

51. Glau CL, Conlon TW, Himebauch AS, Boyer DL, Rosenblatt SA, Nishisaki A. Characterization of thoracic pathophysiologic conditions in patients receiving high-frequency oscillatory ventilation: Pediatric experience. J Ultrasound Med 2018;37(10): 2425-31. doi: 10.1002/jum.14600.

52. Nagano O, Yumoto T, Nishimatsu A, et al. Bias flow rate and ventilation efficiency during adult high-frequency oscillatory ventilation: A lung model study. Intensive care Med Exp 2018;6(1): 11. doi: 10.1186/ s40635-018-0176-3.

53. Hamel DS, Katz AL, Craig DM, Davies JD, Cheifetz IM. Carbon dioxide elimination and gas displacement vary with piston position during high-frequency oscillatory ventilation. Respir Care 2005;50(3): 361-6.

54. High Frequency Oscillating Ventilation (HFOV) - ICU -St George Hospital. 2013; 1-17.

55. Fan E, Villar J, Slutsky AS. Novel approaches to minimize ventilator-induced lung injury. BMC Med 2013;11(1): 85. doi: 10.1186/17417015-11-85.

56. Fan E, Del Sorbo L, Goligher EC, et al. An official American Thoracic Society/European Society of Intensive Care Medicine/Society of Critical Care Medicine clinical practice guideline: Mechanical ventilation in adult patients with acute respiratory distress syndrome. Am J Respir Crit Care Med 2017;195(9): 1253-63. doi: 10.1164/rccm.201703-0548ST.

57. Rose L. High-frequency oscillatory ventilation in adults clinical considerations and management priorities. AACN Adv Crit Care 2008;19(4): 412-20. doi: 10.4037/15597768-2008-4007.

58. Salahuddin N, Al Saidi H, Kherallah M, Solaiman O, Maghrabi K. High frequency oscillatory ventilation may not rescue ARDS patients: An observational study. Crit Care Shock 2013;16(2): 58-64.

59. Sud S, Sud M, Friedrich JO, et al. High-frequency oscillatory ventilation versus conventional ventilation for acute respiratory distress syndrome. Cochrane Database Syst Rev 2016;4: CD004085. doi:10.1002/14651858. CD004085.pub4

60. Meade MO, Young D, Hanna S, et al. Severity of hypoxemia and effect of high-frequency oscillatory ventilation in acute respiratory distress syndrome. Am J Respir Crit Care Med 2017;196(6): 727-33. doi: 10.1164/ rccm.201609-1938OC.

61. Qiao JY, Li YZ, Wang HY, Zhang SD. A Meta analysis of the efficacy of high-frequency oscillatory ventilation versus conventional mechanical ventilation for treating pediatric acute respiratory distress syndrome. CJCP. 2017;19(4): 430-5. doi: 10.7499/j.issn.1008-8830.2017.04.014.

62. De Paoli AG, Clark RH, Bhuta T, Henderson-Smart DJ. High frequency oscillatory ventilation versus conventional ventilation for infants with severe pulmonary dysfunction born at or near term. Cochrane Database Syst Rev 2009;3: CD002974

63. Bhuta T, Clark RH, Henderson-Smart DJ. Rescue high frequency oscillatory ventilation vs conventional ventilation for infants with severe pulmonary dysfunction born at or near term. Cochrane Database Syst Rev 2001;1: CD002974.

64. Wunsch H, Mapstone J, Takala J. High-frequency ventilation versus conventional ventilation for the treatment of acute lung injury and acute respiratory distress syndrome: A systematic review and Cochrane analysis. Anesth Analg 2005;100(6): 1765-72. doi: 10.1213/01.ANE.000014 5070.52315.F2.

65. Shah T. Is there still a role for high-frequency oscillatory ventilation in ARDS? Crit Care Alert 2017;25(8): 1-3. 